\title{
The anti-diabetic drug metformin does not affect bone mass in vivo or fracture healing
}

\author{
J. Jeyabalan • B. Viollet • P. Smitham • S. A. Ellis • \\ G. Zaman • C. Bardin • A. Goodship • J. P. Roux • \\ M. Pierre • C. Chenu
}

Received: 15 October 2012 / Accepted: 8 April 2013 / Published online: 4 May 2013

(C) The Author(s) 2013. This article is published with open access at Springerlink.com

\begin{abstract}
Summary The present study shows no adverse effects of the anti-diabetic drug metformin on bone mass and fracture healing in rodents but demonstrates that metformin is not osteogenic in vivo, as previously proposed.

Introduction In view of the increased incidence of fractures in patients with type 2 diabetes mellitus (T2DM), we investigated the effects of metformin, a widely used T2DM therapy, on bone mass and fracture healing in vivo using two different rodent models and modes of metformin administration.

Methods We first subjected 12-week-old female C57BL/6 mice to ovariectomy (OVX). Four weeks after OVX, mice received either saline or metformin administered by gavage
\end{abstract}

\author{
J. Jeyabalan $\cdot$ S. A. Ellis $\cdot$ G. Zaman $\cdot$ C. Chenu $(\bowtie)$ \\ Department of Comparative and Biomedical Sciences, Royal \\ Veterinary College, Royal College Street, \\ London NW1 0TU, UK \\ e-mail: cchenu@rvc.ac.uk \\ B. Viollet \\ INSERM U1016, Institut Cochin, Paris, France \\ B. Viollet \\ CNRS, UMR8104, Paris, France \\ B. Viollet \\ Université Paris Descartes, Sorbonne Paris Cité, \\ Paris, France \\ P. Smitham • A. Goodship \\ Institute of Orthopaedics \& Musculoskeletal Science, UCL, Royal \\ National Orthopaedic Hospital, Stanmore, UK \\ C. Bardin \\ UF Pharmacocinétique et Pharmacochimie, CHU Cochin AP-HP, \\ Paris, France \\ J. P. Roux $\cdot$ M. Pierre \\ INSERM UMR1033, Université de Lyon, Lyon, France
}

$(100 \mathrm{mg} / \mathrm{kg} /$ daily). After 4 weeks of treatment, bone microarchitecture and cellular activity were determined in tibia by micro-CT and bone histomorphometry. In another experiment, female Wistar rats aged 3 months were given only water or metformin for 8 weeks via the drinking water $(2 \mathrm{mg} / \mathrm{ml})$. After 4 weeks of treatment, a mid-diaphyseal osteotomy was performed in the left femur. Rats were sacrificed 4 weeks after osteotomy and bone architecture analysed by micro-CT in the right tibia while fracture healing and callus volume were determined in the left femur by X-ray analysis and micro-CT, respectively.

Results In both models, our results show no significant differences in cortical and trabecular bone architecture in metformin-treated rodents compared to saline. Metformin had no effect on bone resorption but reduced bone formation rate in trabecular bone. Mean X-ray scores assessed on control and metformin fractures showed no significant differences of healing between the groups. Fracture callus volume and mineral content after 4 weeks were similar in both groups.

Conclusions Our results indicate that metformin has no effect on bone mass in vivo or fracture healing in rodents.

Keywords Bone architecture $\cdot$ Fracture healing · Histomorphometry $\cdot$ Metformin $\cdot$ Micro-CT

\section{Introduction}

Metformin is widely prescribed as a first-line therapy for patients with type 2 diabetes mellitus (T2DM) as an antihyperglycaemic agent which acts primarily by suppressing glucose production by the liver [1]. In contrast to thiazolidinediones (TZD), another T2DM therapy which has adverse effects on the skeleton [2,3], several studies have documented that metformin is osteogenic in vitro. It 
was reported that metformin can induce MC3T3-E1 osteoblastic cells differentiation and bone matrix synthesis via adenosine 5 '-monophosphate-activated protein kinase (AMPK) activation and subsequent induction of endothelial nitric oxide synthase (eNOS) and bone morphogenetic protein-2 (BMP-2) expression [4, 5]. Metformin was also found to regulate Small Heterodimer Partner (SHP) in MC3T3-E1 cells, an orphan nuclear receptor which stimulates osteoblastic bone formation by interacting with the transcription factor Runx2 [6]. Similarly, metformin increased osteoblast proliferation, alkaline phosphatase activity and the number of mineralised nodules formed in rat primary osteoblasts, possibly via stimulation of Runx 2 and IGF-1 production [7, 8]. The action of metformin on bone marrow mesenchymal cell progenitors (BMPCs) has also been investigated and metformin caused an osteogenic effect, suggesting a possible action of metformin in promoting a shift of BMPCs towards osteoblastic differentiation [9]. In contrast, two in vitro studies have shown no effect of metformin on the osteogenic differentiation of bone marrowderived mesenchymal stem cells (MSCs) [10] and matrix mineralisation of both MC3T3-E1 cells and primary osteoblasts [11]. A high concentration of metformin $(2 \mathrm{mM})$ even clearly inhibited osteoblast differentiation [11].

Less work has investigated the effect of metformin on bone in vivo, and the data are more supportive also of an osteogenic effect of metformin. It was reported that 2 months of treatment with metformin prevents the bone loss induced by ovariectomy in rats $[12,13]$, suggesting protective effects of metformin against bone loss. In agreement with these studies, a 2-week treatment with metformin in rats was shown to increase trabecular volume, osteocyte density and osteoblast number in femoral metaphysis [14]. Furthermore, when administered together with the TZD rosiglitazone, metformin prevented the anti-osteogenic effects of rosiglitazone on bone [14]. A very recent study performed in insulin-resistant mice also showed that metformin given for 6 weeks protects femoral bone architecture compared to rosiglitazone, although metformin had no effect on lumbar spine [15]. However, few clinical studies have shown beneficial effects of metformin on bone health. Metformin was shown to reduce the association between diabetes and fractures in human patients [16]. More studies have confirmed that rosiglitazone therapy alone or combined rosiglitazone and metformin therapies were associated with a higher risk of fractures compared to metformin as a monotherapy [17-20]. Interestingly, markers of bone formation were decreased in the metformin group compared to the rosiglitazone one in T2DM patients from the ADOPT study [21].

The aim of our study was to confirm the osteogenic effect of metformin in vivo on bone architecture in basal conditions (control rats) and in osteopenic bone, using a model of bone loss induced by ovariectomy (ovariectomised mice) to mimic the case of post-menopausal women. For each model, we used different modes of metformin administration that have both been utilised in previous rodent studies; while ovariectomised mice had metformin administered orally by gavage, control rats received metformin in the drinking water. We also wanted to explore the hypothesis that metformin promotes fracture healing in a rat model of middiaphyseal, transverse osteotomy in the femur, stabilised via a precision miniature external fixator. Surprisingly, we show in this study that 1- to 2-month treatment with metformin, regardless of the administration route, has no significant effect on rodent bone architecture and fracture healing in vivo, and that metformin significantly reduces bone formation rate in osteopenic trabecular bone.

\section{Materials and methods}

Animals and experimental procedures

Experimental procedures used 3-month-old female Wistar rats (Charles River Laboratories, Inc., Margate, UK) and 3month-old female mice that were in a mixed C57BL/6$129 \mathrm{~Sv}$ genetic background. These mice were bred in our animal facilities and housed in groups of five in polypropylene cages. Wistar rats were allowed to acclimatise for 1 week after transport before the start of experiments and were housed individually. Both rats and mice were subjected to a $12 \mathrm{~h}$ light/dark cycle with room temperature maintained at $21{ }^{\circ} \mathrm{C}$. For mice, metformin (Sigma-Aldrich Company Ltd, Dorset, UK) was given by gavage $100 \mathrm{mg} / \mathrm{kg} /$ daily. For rats, metformin was given in the drinking water at a concentration of $2 \mathrm{mg} / \mathrm{ml}$ for 8 weeks. On average, water consumption in rats is $10-12 \mathrm{ml}$ per $100 \mathrm{~g}$ body weight daily and metformin did not affect the drinking volume. These metformin doses were previously shown to give similar plasma concentrations in rodents than those found therapeutically in humans. The drinking water, along with food, was available ad libitum. The water bottles were replenished twice a week. All animal experimentation procedures were in compliance with Home Office approval and were performed under the threshold of the UK Animals (Scientific Procedures) Act 1986.

Effect of metformin on bone mass in ovariectomised mice

The first experiment was designed to investigate whether metformin could protect against the bone loss induced by ovariectomy. Eighteen female C57BL/6-129Sv mice aged 3 months were all ovariectomised, as previously performed by us $[22,23]$. Four weeks after ovariectomy, mice were divided randomly into two groups, one $(n=9)$ receiving 
saline while the other one $(n=9)$ receiving metformin $(100 \mathrm{mg} / \mathrm{kg})$ daily by gavage for 4 weeks. At days 6 and 3 prior to euthanasia, mice were intraperitoneally injected with calcein (Sigma-Aldrich) and alizarin red complexone (Sigma-Aldrich), respectively, to label bone-forming surfaces in trabecular bone. At the end of the experiment, mice were sacrificed, the serum collected for measurement of metformin concentration, the tibia dissected for micro-CT analysis of cortical and trabecular bone parameters and bone histomorphometry while the femora were used for protein isolation and RT-PCR analysis. Since we did not have a SHAM group, the success of ovariectomy was evaluated by uterine atrophy observations during dissection.

Effect of metformin on bone mass and fracture healing in rats

The second experiment was designed to investigate the effect of metformin on basal bone mass. For this study, we used the right contra-lateral tibia of non-ovariectomised female rats which underwent a fracture in the left femur. Twenty female Wistar rats of approximately $200 \mathrm{~g}$ were divided randomly into two groups, one $(n=10)$ having access to drinking water alone while the other $(n=10)$ receiving metformin in the drinking water $(2 \mathrm{mg} / \mathrm{ml})$ for 8 weeks. Four weeks after the beginning of treatment, all the rats $(n=20)$ underwent a middiaphyseal transverse osteotomy in the left femur as described previously [24]. Surgery was performed under general anaesthesia (ketamine $75 \mathrm{mg} / \mathrm{kg}$ and xylazine $10 \mathrm{mg} / \mathrm{kg}$ ) and appropriate gaseous anaesthesia using aseptic techniques. The external fixator system used in this protocol comprises two metal blocks of titanium alloy linked to two cylindrical stainless steel bars. Briefly, the fixator was applied to the craniolateral aspect of the femur using four threaded M1.2 stainless steel pins. Consistent positioning of the fixator pins was ensured using a drill locator template. After pin placement, a transverse osteotomy was created midway between the proximal and distal pins using an oscillating diamond bone saw, with saline irrigation throughout. The bone fragments were distracted to leave an osteotomy gap of $0.5 \mathrm{~mm}$ that was maintained by locking the fixator blocks on to the connecting bars. The rats were administered with $0.1 \mathrm{cc}$ of Vetergesic (Alstoe Ltd, York, UK) for analgesia and $0.05 \mathrm{cc}$ of cephalosporin (Sandoz Ltd, Camberley, UK), as a single dose to prevent infection, postoperatively and were returned to their cages. They were granted mobility immediately after regaining consciousness. Radiographs of the operation site were taken at 4 weeks postfracture, the time where rats were euthanised under anaesthesia via the delivery of $\mathrm{CO}_{2}$ into an inhalation chamber. Right tibiae were collected for micro-CT analysis of cortical and trabecular bone parameters while left osteotomised femora were collected for micro-CT analysis of fracture callus and histology.
Micro-CT analysis of mouse and rat tibiae

Right tibiae were harvested from 5-month-old OVX female C57BL/6-129Sv mice, fixed in $10 \%$ neural-buffered formalin for $24-72 \mathrm{~h}$ and stored in $70 \%$ ethanol at $4{ }^{\circ} \mathrm{C}$. These tibia were then scanned with high-resolution $(5 \mu \mathrm{m}$ pixel size) micro-computed tomography (micro-CT, SkyScan 1172; SkyScan, Kontich, Belgium), as previously described [7]. Right tibiae from the fracture study were dissected from rats, fixed and stored as above and scanned with a lower resolution of $14 \mu \mathrm{m}$ pixel size due to the size of the bones. The whole tibiae were reconstructed using NRecon v.1.4.4.0 (SkyScan) and bone histomorphometric analyses in two and three dimensions (2D, 3D) were performed by SkyScan software (CT-Analyser v.1.5.1.3). For the analysis of trabecular bone, the cortical shell was excluded by operator-drawn regions of interest and $3 \mathrm{D}$ algorithms were used to determine the relevant parameters which included bone volume percentage (BV/TV), trabecular thickness, trabecular number, trabecular spacing, structure model index (SMI), trabecular pattern factor and degree of anisotropy. Analysis of cortical bone was performed using a 0.49 -mm-long segment (or 100 tomograms) at $37 \%$ of the tibias' length from its proximal end. For analysis of the cortical bone compartment, 2D computation was used and parameters were determined for each one of the 100 tomograms and then averaged. They included periosteal perimeter, endosteal perimeter and cortical thickness.

\section{Assessment of fracture healing}

\section{$X$-ray analysis}

Radiographs were taken at the study end point (8 weeks), prior to euthanasia. Both dorsal and ventral X-rays were performed to assess the extent of in situ healing and bridging of the fracture space. Fracture healing was scored from two dimensions, anterior-posterior and lateral-medial X-rays. The X-rays were scored using a three-point system, $1-$ no callus, 2-some callus formation and 3-significant callus, on all four cortices. The lowest score is thus 4 , signifying no callus formation on all four cortices, and a highest of 12 , significant callus growth in all four regions.

\section{Micro-CT analysis of fracture healing}

Left femora (fractured side) were scanned at $14 \mu \mathrm{m}$ resolution using micro-CT (SkyScan 1172). A length of approximately $15 \mathrm{~mm}$ of the callus with the fracture in the centre was scanned. Histomorphometric analysis of fracture callus in 2D and 3D was performed by SkyScan software (v. 1.11.8.0). A 'shrink-wrap' algorithm was used to define the tissue perimeter as the volume of interest (VOI). 
Binarisation of the reconstructed datasets was by two methods that applied different thresholds since the fracture callus 4 weeks after fracture is heterogeneous and may contain low or highly mineralised woven bone; to automatically delineate the low mineralised callus, a specific threshold was applied that excluded the highly mineralised callus and cortical bone. After measurement, another thresholding was applied, which in contrast defined highly mineralised callus and cortical bone, excluding the very low mineralised callus. Two relevant parameters were therefore quantified, cortical and mineralised callus volume and low mineralised callus volume.

\section{Histology}

After micro-CT analysis, fracture calluses were decalcified in $0.34 \mathrm{M}$ EDTA in PBS for 2 weeks at room temperature, bisected longitudinally and the lateral half embedded in paraffin as described previously [25]. Sagittal sections $(5 \mu \mathrm{M})$ were cut from the paraffin blocks using a microtome (HM360; Fisher Scientific UK Ltd, Loughborough, UK). Sections were stained with haematoxylin and eosin (H\&E) for basic morphology and with Alcian blue and nuclear fast red for analysis of cartilage and bone.

\section{Histomorphometry analysis of tibia}

Tibia were fixed in $10 \%$ neutral-buffered formalin for $24 \mathrm{~h}$, dehydrated and embedded in methyl methacrylate (MMA) at low temperature to preserve enzymatic activity [26]. Unstained $8-\mu \mathrm{m}$-thick sections were used for fluorescence microscopy to assess mineral apposition rate (MAR, $\mu \mathrm{m} /$ day). Mineralising surfaces were expressed as alizarin red-labelled surfaces per bone surfaces (MS/BS, \%) and the bone formation rate was calculated as $\mathrm{MS} / \mathrm{BS} \times \mathrm{MAR}\left(\mathrm{BFR} / \mathrm{BS}, \mu \mathrm{m}^{3} / \mu \mathrm{m}^{2} /\right.$ day) [27]. Alternatively, sections were stained for tartrate-resistant acid phosphatase (TRAP) (Leucognost ${ }^{\circledR}$ SP; Merck, Germany) and counterstained with Weigert haematoxylin solution. Histomorphometric parameters were measured on the trabecular bone of the metaphysis, on a region of interest consisting of $2 \mathrm{~mm}$ width below the growth plate. Measurements were performed using an image analysis software (Tablet'measure; Explora Nova, La Rochelle, France). Histomorphometric parameters were reported in accordance with the ASBMR Committee nomenclature [28].

Protein extraction and western blot analysis

For the isolation of total proteins, right femora from 5-monthold female C57BL/6-129Sv mice were carefully dissected and all their surrounding musculature removed leaving the periosteum intact. We also dissected femora from wild-type
C57BL/6 mice that were injected with metformin at $100 \mathrm{mg} / \mathrm{kg} /$ daily only for 3 days. The cartilaginous ends of the bones were separated and the remaining femoral shafts were flushed with PBS to remove the marrow. The femoral shafts were then snap-frozen and pulverised under liquid nitrogen using a mortar and pestle, and then lysed in cold denaturing lysis buffer $(2 \%$ SDS, $2 \mathrm{M}$ urea, $8 \%$ sucrose, $20 \mathrm{mM}$ sodium glycerophosphate, $1 \mathrm{mM}$ sodium fluoride and $5 \mathrm{mM}$ sodium orthovanadate). Proteins were denatured by boiling for $10 \mathrm{~min}$ and concentrations determined by BCA protein assay. Twenty micrograms of proteins was size-fractionated using SDS-PAGE and electrotransferred onto Protran nitrocellulose membranes (Schliecher and Schuell, Dassel, Germany). Membranes were blocked for $1 \mathrm{~h}$ in $0.2 \%(\mathrm{w} / \mathrm{v})$ I-block (Topix, Bedford, MA, USA) before being incubated with primary antibodies. The blots were incubated overnight at $4{ }^{\circ} \mathrm{C}$ with antibodies against total AMPK $\alpha 1 / 2$ (tAMPK $\alpha 1 / 2$, rabbit), phospho-(Thr-172)AMPK $\alpha 1 / 2$ (pAMPK $\alpha 1 / 2$, rabbit) (New England Biolabs, Hitchin, UK) and $\beta$-actin (goat) (Dako, Ely, UK), all added at a 1:1,000 dilution. The following secondary antibodies were used, goat anti-rabbit (New England Biolabs) against tAMPK and pAMPK $1 \alpha 1 / 2$ and rabbit anti-goat (Dako) against $\beta$-actin antibody, both at 1:2,500 dilution at room temperature for $1 \mathrm{~h}$. Proteins were visualised using the enhanced chemiluminescence detection system (ECL) (GE Healthcare UK Ltd, Little Chalfont, UK). The intensity of the specific bands was quantified by densitometry using Image J software.

\section{RNA extraction and quantitative real-time PCR}

Total RNA was isolated from left whole femora after removal of the bone marrow, as previously described [7]. RNA from three femora in each treatment group was pooled and two separate extractions were performed. Total RNA was reverse-transcribed with Superscript II reverse transcriptase. Real-time QPCR was carried out as described earlier [29] using QuantiTect SYBR green PCR kit and Opticon 2 LightCycler (MJ Research, Waltham, MA, USA). Primer sequences were obtained from Qiagen and are summarised in Table 1. The expression levels for Osterix and Runx 2 were normalised to the reference gene $18 \mathrm{~s}$ rRNA.

Plasma quantification of metformin

Concentrations of basal metformin level in plasma were determined using a modified ultra high-pressure liquid chromatography (UHPLC) assay with UV DAD (diode array detector) as initially described [30]. Liquid-liquid extraction of metformin was performed as follows: $200 \mu \mathrm{l}$ of plasma sample was buffered by adding $200 \mu \mathrm{l}$ of $8 \mathrm{M}$ sodium hydroxide and spiked with $40 \mu \mathrm{l}$ phenylbiguanide (internal standard). Then $2.6 \mathrm{ml}$ of a mixture of 50:50 
Table 1 Quantitative real-time RT-PCR primer sequences $\left(5^{\prime} \rightarrow 3^{\prime}\right)$

\begin{tabular}{llllcc}
\hline Gene & Sequence (forward) & Sequence (reverse) & Position & Length (bp) & PrimerBank IDs \\
\hline Runx2 & GACTGTGGTTACCGTCATGGC & ACTTGGTTTTTCATAACAGCGGA & $474-557$ & 84 & $225690525 \mathrm{~b} 1$ \\
Osterix & ATGGCGTCCTCTCTGCTTG & TGAAAGGTCAGCGTATGGCTT & $1-156$ & 156 & $18485518 \mathrm{a} 1$ \\
$18 \mathrm{~s}$ & GTAACCCGTTGAACCCCATT & CCATCCAATCGGTAGTAGCG & $5231-5381$ & 151 & NR_003286.2 \\
\hline
\end{tabular}

1-butanol $/ n$-hexane was added, the mixture centrifuged and $200 \mu 1$ of $1 \%$ acetic acid was added to the upper organic layer. The mixture was centrifuged, the upper organic layer discarded and $5 \mu \mathrm{l}$ of the aqueous layer was then injected onto a Kinetex ${ }^{\circledR}$ Hilic column $(100 \times 4.6 \mathrm{~mm}$ ID, $2.6 \mu \mathrm{m})$ maintained at $40{ }^{\circ} \mathrm{C}$. Flow rate was set $1 \mathrm{ml} / \mathrm{min}$ and compounds were detected at $234 \mathrm{~nm}$ on an Agilent DAD (1260 Infinity ${ }^{\circledR}$ ). Retention times for phenylbiguanide and metformin were respectively 3.0 and $4.5 \mathrm{~min}$. Lower limit of quantification was $15 \mathrm{ng} / \mathrm{ml}$. Based on quality control samples, intraday and between-days precision and accuracy were less than $10 \%$ over the entire range of quantification.

\section{Statistics}

The results were presented as mean values \pm SD. Statistical analysis was performed using a two-tailed Mann-Whitney $U$ test with GraphPad Prism software. $P$ values less than 0.05 were considered to be statistically significant.

\section{Results}

Metformin has no effect on in vivo bone loss induced by ovariectomy in mice

To investigate the effect of metformin on the bone loss induced by ovariectomy in tibia, we subjected 12-weekold female C57BL/6-129Sv mice to ovariectomy (OVX) and metformin treatment by gavage for 4 weeks. To confirm that metformin treatment administered by gavage was effective, we assessed metformin concentration in plasma and showed its detection solely in the plasma of the treatment group (Fig. 1a). Four weeks of treatment with metformin induced a trend for total body weight loss in mice, although this did not reach statistical significance (Fig. 1b). Visceral and subcutaneous fat weights were not modified by metformin treatment (Fig. 1c).

The analysis of bone micro-architecture determined by micro-CT in tibia of metformin-treated OVX mice showed no significant changes in both the trabecular and cortical compartments compared to control mice (Fig. 2). Metformin had no effect on trabecular bone volume (BV/TV), trabecular number and thickness compared to saline (Fig. 2a-c). Other trabecular parameters such as trabecular separation, bone pattern factor, degree of anisotropy and SMI (not shown) were also not statistically different between salineand metformin-treated mice. Similarly, metformin had no significant effect on cortical thickness and periosteal and endosteal perimeters (Fig. 2d-f).

Metformin decreases bone formation parameters in ovariectomised mice

We examined bone cellular activities in the tibia of ovariectomised mice using bone histomorphometry. Analysis of bone formation rate using double fluorescence labelling showed that metformin decreases the mineralising surfaces and MAR compared to control mice (MS/BS - metformin, $44.19 \pm 15.1 \%$ vs. control, $56.38 \pm 7.13 \%, P=0.14$; MARmetformin $1.25 \pm 0.14 \mu \mathrm{m} /$ day vs. control, $1.38 \pm 0.16 \mu \mathrm{m} /$ day, $P=0.2$ ) and significantly reduces the bone formation rate (Fig. 3a) (BFR - metformin, $0.543 \pm 0.168 \mu \mathrm{m}^{3} / \mu \mathrm{m}^{2} /$ day vs. control, $0.778 \pm 0.116 \mu \mathrm{m}^{3} / \mu \mathrm{m}^{2} /$ day, $\left.P=0.02\right)$. The percentage of TRAP positive surfaces (osteoclast surfaces) was not different in the metformin-treated mice compared to control mice (metformin, $5.93 \pm 2.29 \%$ vs. control, $5.01 \pm 2.18 \% ; P=0.31$ ) (Fig. 3b).

Metformin has no effect on bone mass in vivo in rats

To analyse the effect of metformin on bone mass in vivo, we submitted 3-month-old female Wistar rats to metformin treatment during 8 weeks. In this experiment, metformin was given in the drinking water, a mode of administration which has been previously shown to be effective in rats at this concentration [31]. Metformin did not significantly affect rat body weight after 8 weeks of treatment (metformin, $223.4 \pm 14.1 \mathrm{~g}$ vs. saline, $232.8 \pm 16.6 \mathrm{~g}, P=0.1$ ). Our micro$\mathrm{CT}$ analysis of tibia from saline- and metformin-treated rats showed no significant effect of metformin on bone trabecular (Fig. 4a-c) and cortical parameters (Fig. 4d-f). Metformin induced a non-significant increase in $\mathrm{BV} / \mathrm{TV}$, trabecular number and trabecular thickness (Fig. 4a-c). Trabecular separation was decreased by metformin treatment, but it was not significant (metformin, $0.16 \pm 0.01$ vs. saline, $0.18 \pm 0.01$, $P=0.1$ ), as well as SMI (metformin, $0.69 \pm 0.32$ vs. saline, $1.28 \pm 0.15, P=0.2$ ) and trabecular bone pattern factor (metformin, $-0.27 \pm 2.7$ vs. saline, $4.34 \pm 2.07, P=0.2$ ). Metformin had no effect on the cortical parameters (Fig. $4 \mathrm{~d}-\mathrm{f}$ ). 

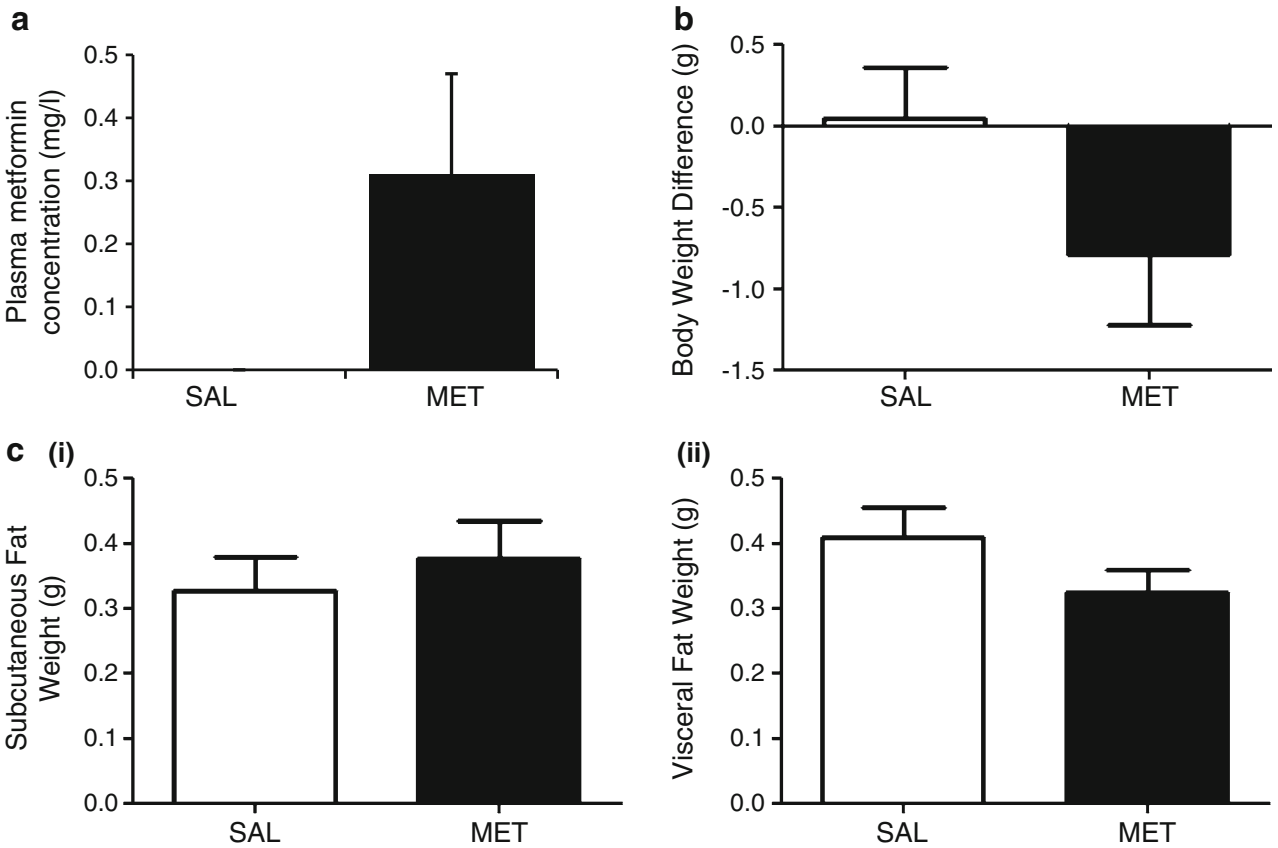

Fig. 1 Effect of metformin treatment on plasma metformin concentration, body and tissue weights in ovariectomised mice. a Metformin concentration was quantified by HPLC analysis in plasma of all mice after 4 weeks of treatment with saline and metformin. b Body weight difference between start and end of metformin treatment period in

ovariectomised wild-type mice. c Weights of $i$ subcutaneous fat and ii visceral fat after 4 weeks of treatment with saline and metformin in ovariectomised wild-type mice. Bars represent mean \pm SD of $n=9$ mice/group
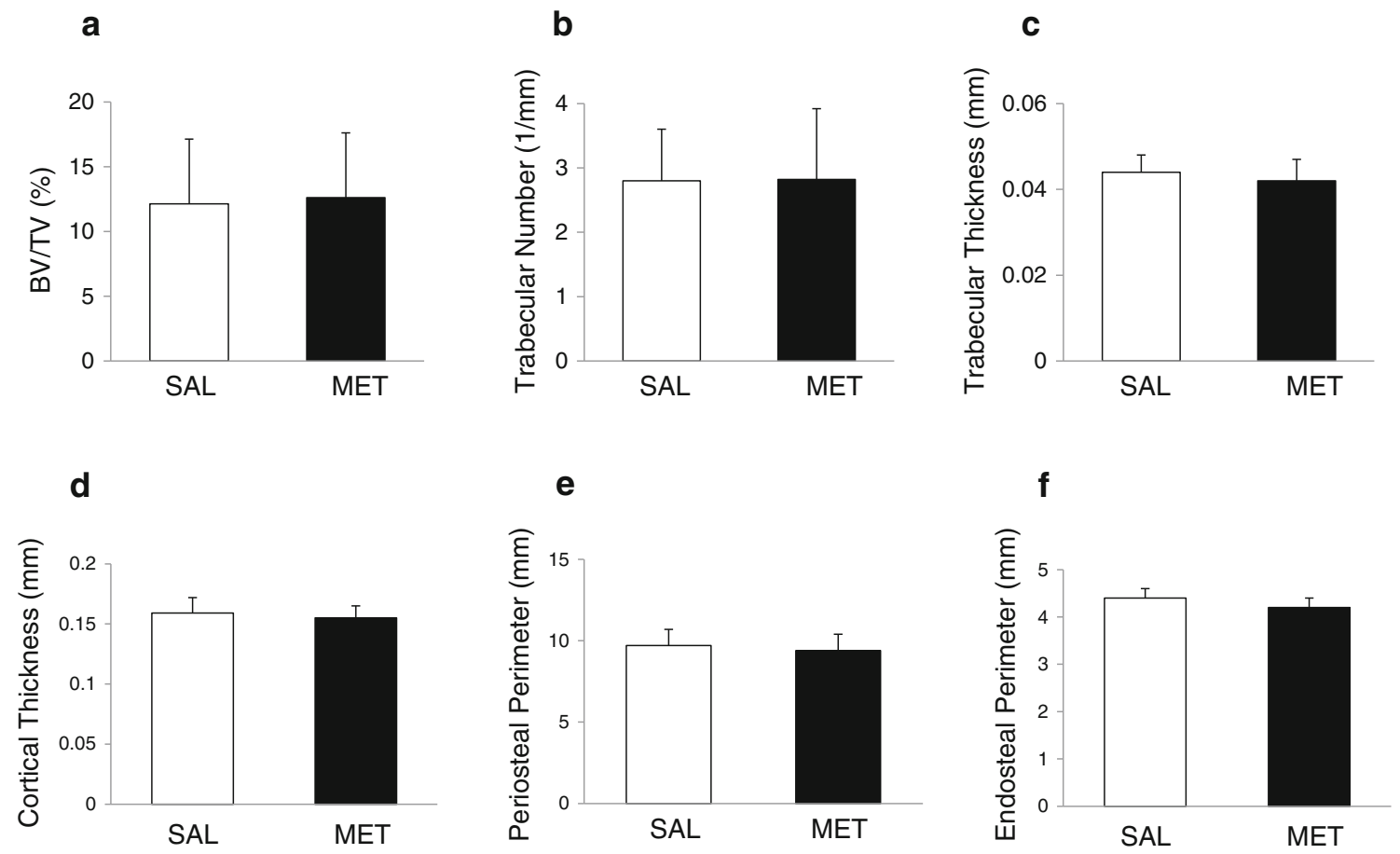

Fig. 2 Effect of metformin treatment on trabecular and cortical bone parameters in tibia of 5-month-old ovariectomised wild-type mice. a, b, c Three-dimensionally computed BV/TV (a), trabecular number (b) and trabecular thickness (c) were assessed by micro-CT in the proximal tibial metaphysis of saline- and metformin-treated mice. d, e, f Two-

dimensionally computed cortical thickness (d), periosteal perimeter (e) and endosteal perimeter (f) were assessed by micro-CT in the mid-diaphysis of cortical bone in saline- and metformin-treated mice. Bars represent mean \pm $\mathrm{SD}$ of $n=9$ mice/group 
a
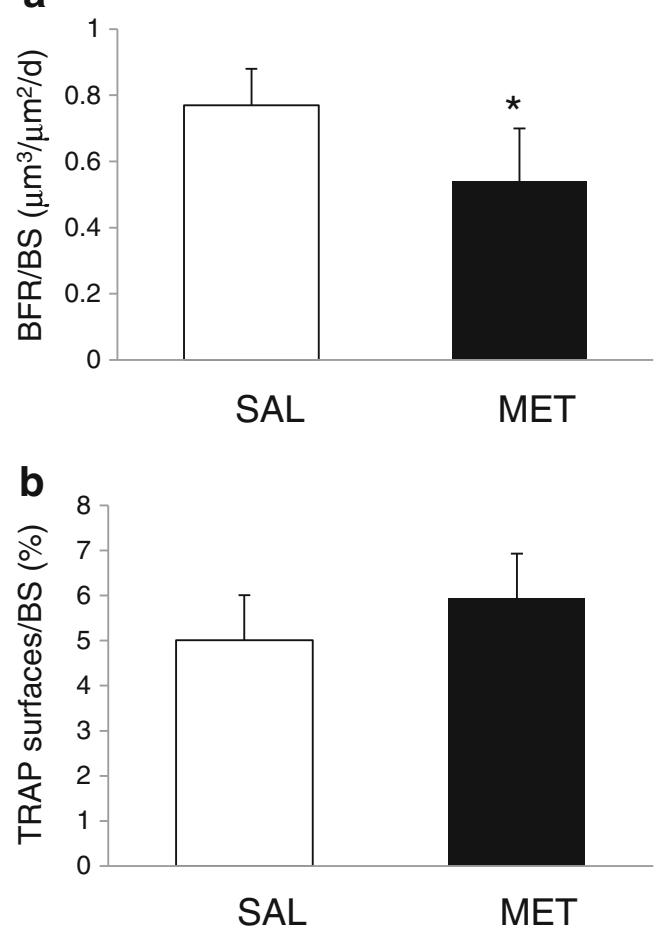

Fig. 3 Effect of metformin treatment on bone histomorphometry parameters measured in tibia of 5-month-old ovariectomised wild-type mice. a Bone formation rate $(B F R)$ measured on trabecular region of mouse tibia sections labelled with calcein and alizarin red from salineand metformin-treated mice. b Percentage of TRAP-stained surfaces/ bone surfaces in trabecular region of mouse tibia sections from salineand metformin-treated mice. Values are mean $\pm \mathrm{SD}$ of $n=6 / 7$ mice/ group, $* P=0.02$

Metformin has no effect on fracture healing after 4 weeks

We evaluated the effect of metformin treatment on fracture healing in rats 4 weeks after fracture. Radiography showed that not all fractures were united after 4 weeks. We had to exclude three rats due to fractures at the pin site and wound dehiscence decreasing the total number of rats to 17 . The final number of rats for each group was eight in the control group and nine in the metformin group. To assess the state of fracture healing, Xray scoring was carried out on four cortices using radiographic images. Mean X-ray scores of both control and metformintreated groups showed no significant differences between groups (Fig. 5a). Representative 3D views of callus structure for both groups are illustrated in Fig. 5c. Large periosteal calluses are visible at the fracture site in both the control and metformin-treated groups. Data for fracture callus volumes are shown in Fig. 5b. Volumes of both low mineralised callus and highly mineralised callus and cortical bone were similar between control and metformin groups, suggesting that metformin treatment does not affect fracture callus size or speed of healing. Figure $5 \mathrm{~d}$ shows representative images of H\&E- and Alcian blue-stained fracture calluses at 4 weeks in saline and metformin-treated groups. The original cortical bone and site of fracture are evident. The callus of both groups contained cartilage as demonstrated by Alcian blue staining and small regions of primary trabecular-like bone throughout the callus area. Metformin did not affect the progression of endochondral ossification and fracture healing 4 weeks after osteotomy, confirming the micro-CT data (Fig. 5d).

Metformin does not activate AMPK in bone nor regulate expression of osteoblast-specific transcription factors

Since AMPK activation has been shown to be important for osteogenesis [7] and is involved in metformin's mechanism of action [32], we studied the involvement of AMPK activation in its effects on bone. We found that short-term treatment (3 days) of C57BL/6 wild-type mice with metformin stimulates AMPK phosphorylation in liver while having no effect on AMPK phosphorylation in bone (Fig. 6a). Our results also show no significant increase in AMPK phosphorylation in femora and fat of ovariectomised C57BL/6-129Sv mice after 4 weeks of treatment with metformin (Fig. 6b). These results indicate that AMPK is not activated by short and prolonged metformin treatment in bone. We did not detect any difference in Osterix and Runx2 expressions in femora between the saline and metformin groups after 4 weeks treatment (Fig.6c), indicating that metformin does not activate osteoblast-specific gene markers.

\section{Discussion}

With the increasing worldwide prevalence of T2DM which predisposes patients to osteoporosis and increased risk of fractures [33,34], there is an increasing need to evaluate the skeletal actions of anti-diabetic drugs and to examine their effects on healing of osteoporotic fractures. We show in this study that the anti-diabetic drug metformin is not 'bone unfriendly' but has no osteogenic action, as previously reported. In contrast, our data indicate that metformin reduces bone formation rate, has no major effect on bone mass in vivo in rodents and does not promote fracture healing.

We first used ovariectomised mice to examine the skeletal effect of metformin in conditions of low bone mass that are more representative of the frequent secondary osteoporosis observed in T2DM patients. Our results, which show no effect of metformin on bone architecture, contrast with two previous studies performed in ovariectomised rats [12, 13], demonstrating that metformin inhibits the trabecular bone loss [12] and the decrease in bone mineral density [13] induced by OVX. In both studies, metformin was also administered to OVX rats by gavage at an identical concentration with the one used in our work. Although we did not perform a dose-response of metformin in our study, the concentration of metformin given orally has been extensively used in previous rodent studies $[35,36]$. Our metformin treatment was efficient since plasma levels of metformin were 
a

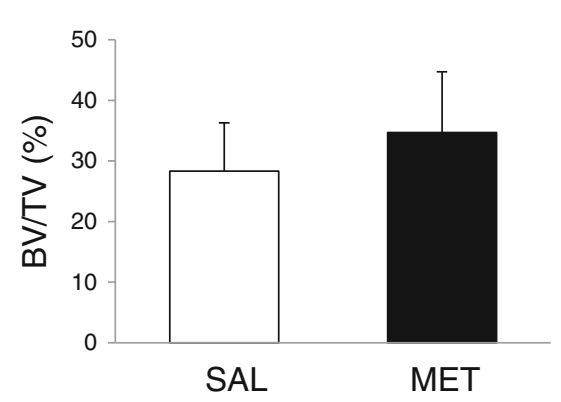

d

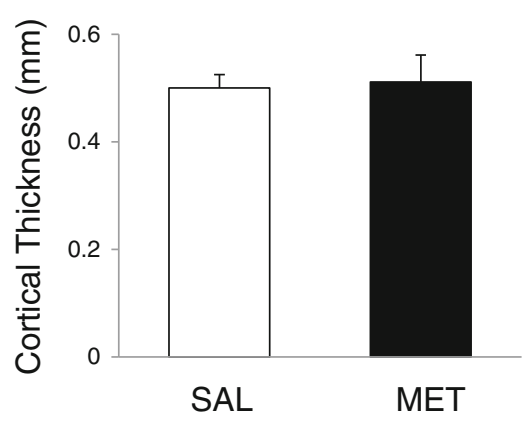

b

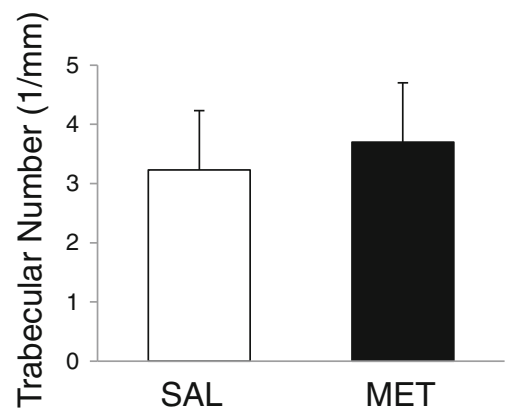

e

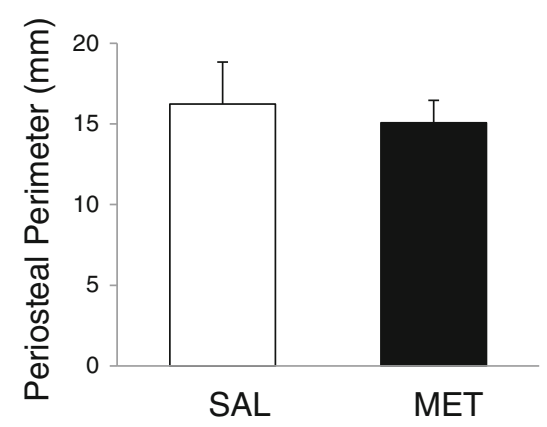

C
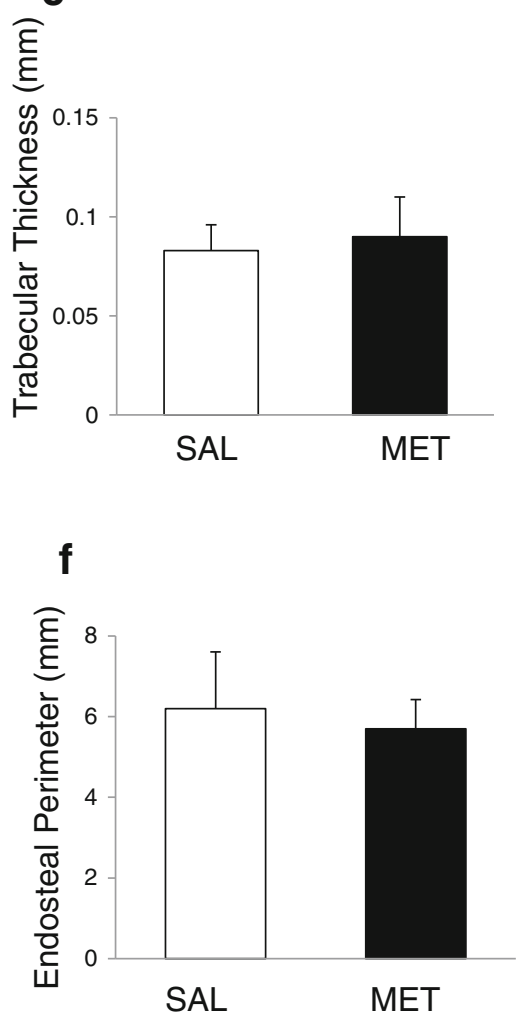

Fig. 4 Effect of metformin on trabecular and cortical bone parameters in rat tibia aged 5 months treated with saline and metformin during 8 weeks. a, b, c Three-dimensionally computed BV/TV (a), trabecular number (b) and trabecular thickness (c) were assessed by micro$\mathrm{CT}$ in the proximal tibial metaphysis of saline- and metformin-treated

rats. d, e, f Two-dimensionally computed cortical thickness (d), periosteal perimeter (e) and endosteal perimeter (f) were assessed by micro-CT in the mid-diaphysis of cortical bone in saline- and metformin-treated rats. Bars represent mean $\pm \mathrm{SD}$ of $n=9$ rats/group

detected with a value of approximately $0.3 \mathrm{mg} / \mathrm{l}$. In addition, metformin induced a small decrease in body weight in our study, a known effect of this anti-diabetic drug which promotes satiety, reducing the food intake [37]. It is therefore difficult to reconcile our data with these previous rat studies, all the more since Gao's study [12] showed similar trabecular bone mass to ours after OVX and we previously showed that same-age OVX mice on this C57BL/6-129Sv background can experience large increases in trabecular bone volume when treated with intermittent PTH [23]. The duration of metformin treatment is unlikely to explain those differences since we treated our mice with metformin for 1 month, but our rats for 2 months, similarly to the previous rat studies. The effects of metformin on bone may however vary depending on the rodent species and strain utilised, as previously demonstrated for the skeletal effect of rosiglitazone [38, 39]. In our second study, we used non-OVX rats to examine the effect of metformin on basal bone mass. Rats were used as we wanted to utilise the non-fractured legs of our model of mid-diaphyseal, transverse osteotomy in the rat femur. Metformin was given this time in the drinking water as this mode of administration is less stressful than gavage for fracture experiments and also widely used. Similarly, we found no effect of metformin on bone architecture in contrast to a recent publication by Sedlinsky et al. [14] showing by histology analysis that metformin increases trabecular area when administered to non-OVX adult rats for 2 weeks in the drinking water, at similar concentration, but in a different strain of rats. Although trabecular and cortical bone architectural parameters were not measured in this study using micro-CT, osteoblast numbers and resorption surfaces were quantified on paraffin sections and were both stimulated by metformin treatment, suggesting that metformin increases bone remodelling in favour of formation [14]. In our mouse study, dynamic bone parameters measurements were performed in undecalcified sections of tibiae, and we found that osteoclast surfaces were not affected by metformin treatment. In addition, we showed that the dynamic measure of bone formation, BFR, was significantly decreased in trabecular bone by metformin. This resulted from reduction of both MAR and MS/BS which reflects decreased osteoblast number and activity, although these two parameters of bone formation, when independent, were not decreased significantly with metformin treatment. The demonstration that metformin has no resulting effect on trabecular bone architecture, despite inducing a significant decrease in BFR in trabecular bone, could suggest other indirect effects of 


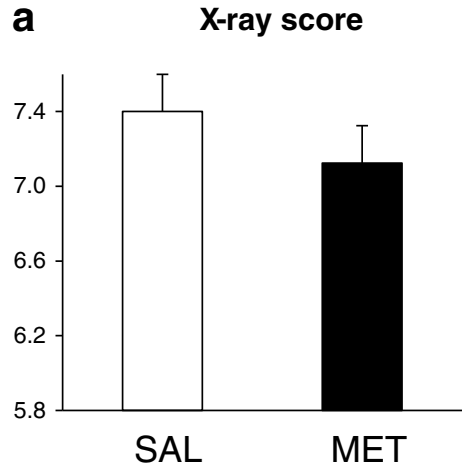

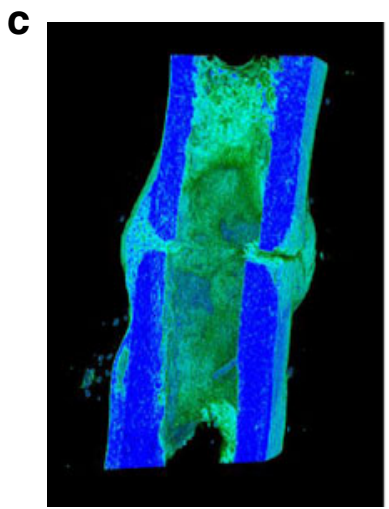

SAL

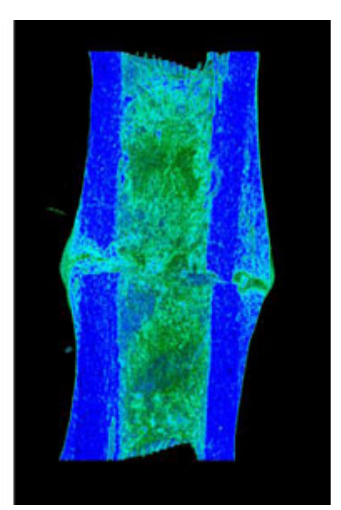

MET b (i) Percentage of highly mineralised callus and bone per tissue volume

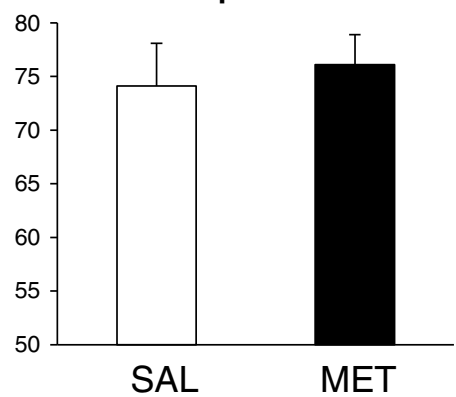

(ii) Percentage of low mineralised

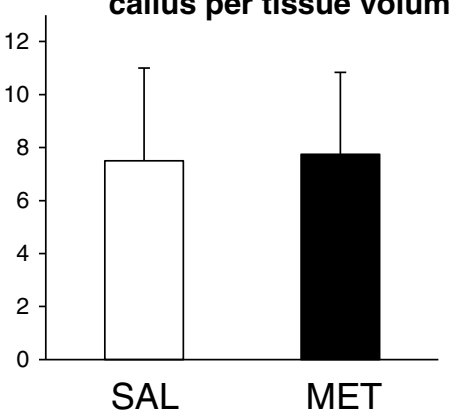
callus per tissue volume

SAL d

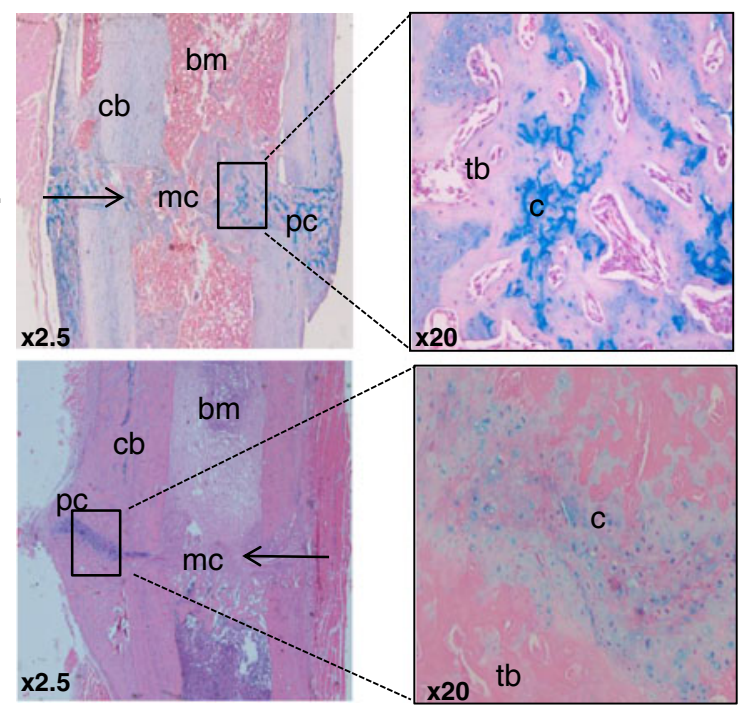

Fig. 5 Effect of metformin on bone fracture healing. a X-ray scoring results for fractured femora in control and metformin-treated rats 4 weeks after fracture. b Analysis of the reconstructions of the fracture callus using the 3D SkyScan software. The volumes of highly mineralised callus and bone $(i)$ and low mineralised callus (ii) are not significantly different in control and metformin-treated groups. Bars represent mean $\pm \mathrm{SD}$ of $n=8 / 9$ rats/group. c Representative reconstructed $3 \mathrm{D}$ images of rat fracture callus in control and metformin-treated groups. The dark blue colour represents cortical bone and

metformin, possibly affecting osteoblastogenesis. These results are in agreement with the demonstration that markers of osteoblast activity were reduced for women and men in the metformin group compared to the rosiglitazone one in T2DM patients from the ADOPT study [21]. However, similarly to Wang's study [15], our preliminary results did not demonstrate changes in expression of osteoblast-specific transcription factors measured by quantitative RT-PCR in metformin-treated bones compared to control ones. The discrepancies between all these in vivo studies may therefore also arise from the fact that they measured diverse bone and cellular parameters.

Studies that have investigated the in vitro effects of metformin on bone have also shown discrepancies. While the majority of studies reported osteogenic effects of metformin in vitro $[4-9,40]$, there are reports indicating that highly mineralised callus and the bluish green colour trabecular bone and low mineralised callus. d H\&E- and Alcian blue-stained longitudinal sections of fracture callus in control and metformin-treated rats. At 4 weeks, fractures appeared mostly bridged and the overall fracture callus size in the two groups was the same. There was also no obvious visible difference in bone and cartilage composition in control and metformin-treated groups, as shown by Alcian blue staining. Right arrow fracture gap, $b m$ bone marrow, $c b$ cortical bone, $p c$ periosteal callus, $m c$ medullary callus, $c$ cartilage, $t b$ trabecular-like bone blast differentiation [11]. Metformin was also shown to inhibit osteoclast differentiation in vivo and in vitro by stimulating osteoprotegerin and inhibiting RANKL expressions [13, 41], although Bak et al. [40] showed no effect of metformin on osteoclast formation. Few clinical studies in diabetic patients have assessed the effect of metformin as a monotherapy on fracture risk, and they show overall poor evidence that it has major anabolic effects on bone. No direct links between metformin and falls [42] were demonstrated, and data regarding the association of metformin with fracture risk are unclear $[16,43,44]$. Borges et al. [45] have recently shown that 80 weeks of metformin treatment in drug-naïve T2DM patients induces very modest increases in lumbar spine and total hip BMD. However, metformin has no osteogenic effect [10] or inhibits osteo- 
a (i)

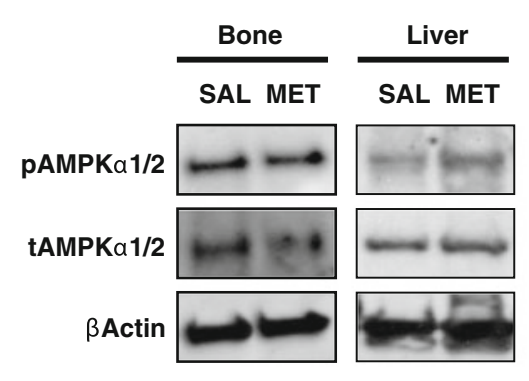

b (i)

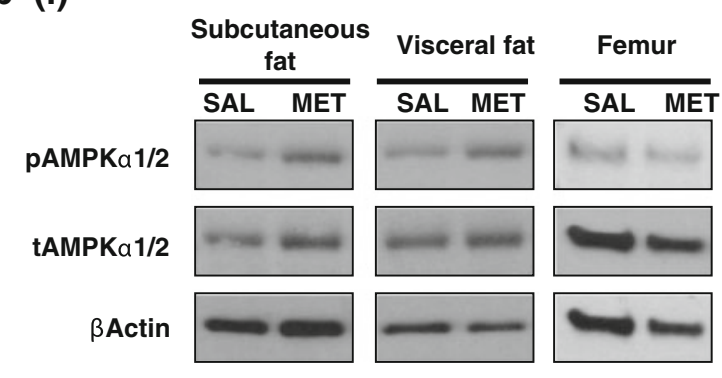

C

\begin{tabular}{lll} 
& \multicolumn{2}{c}{ Normalised mRNA expression levels } \\
\cline { 2 - 3 } & Osterix & Runx2 \\
Saline & $1.95 \mathrm{E}-07$ & $1.47 \mathrm{E}-05$ \\
Metformin & $1.12 \mathrm{E}-07$ & $1.03 \mathrm{E}-05$ \\
& & \\
Saline & $1.56 \mathrm{E}-06$ & $3.19 \mathrm{E}-05$ \\
Metformin & $2.68 \mathrm{E}-06$ & $5.26 \mathrm{E}-05$
\end{tabular}

Fig. 6 Effect of metformin treatment on AMPK $\alpha$ phosphorylation in bone. a, $i$ Western blot analysis of pAMPK $\alpha 1 / 2$, tAMPK $\alpha 1 / 2$ levels in bone and liver after 3 days of treatment with metformin $(100 \mathrm{mg} / \mathrm{kg})$. Representative immunoblots are shown, repeated with similar results twice; $\mathbf{a}, i i$ all blots were quantified using image $\mathrm{J}$ and the pAMPK to tAMPK ratio relative to $\beta$-actin was determined for each experiment. Bars represent mean $\pm \mathrm{SD}, n=4$ biological samples $* P<0.05$. b, $i$ Western blot analysis of pAMPK $\alpha 1 / 2$, tAMPK $\alpha 1 / 2$ levels in subcutaneous and visceral fat depots and in femur of ovariectomised wild-type

metformin treatment was recently shown to decrease circulating sclerostin levels in men with T2DM [46], suggesting that it could improve skeletal fragility in those patients. More clinical studies have compared the effects of combined TZDs and metformin therapies to TZDs alone and have more consistently shown that metformin decreases fracture risk compared to TZDs [17-20].

Metformin is an AMPK agonist [32, 47], and our previous work has established that AMPK is important for bone mass in vivo [7, 23]. The contribution of AMPK to the skeletal action of metformin is unknown. Our results demonstrate that both 3-day and 1-month treatments with metformin did not stimulate AMPK phosphorylation in bone in

(ii)

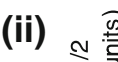
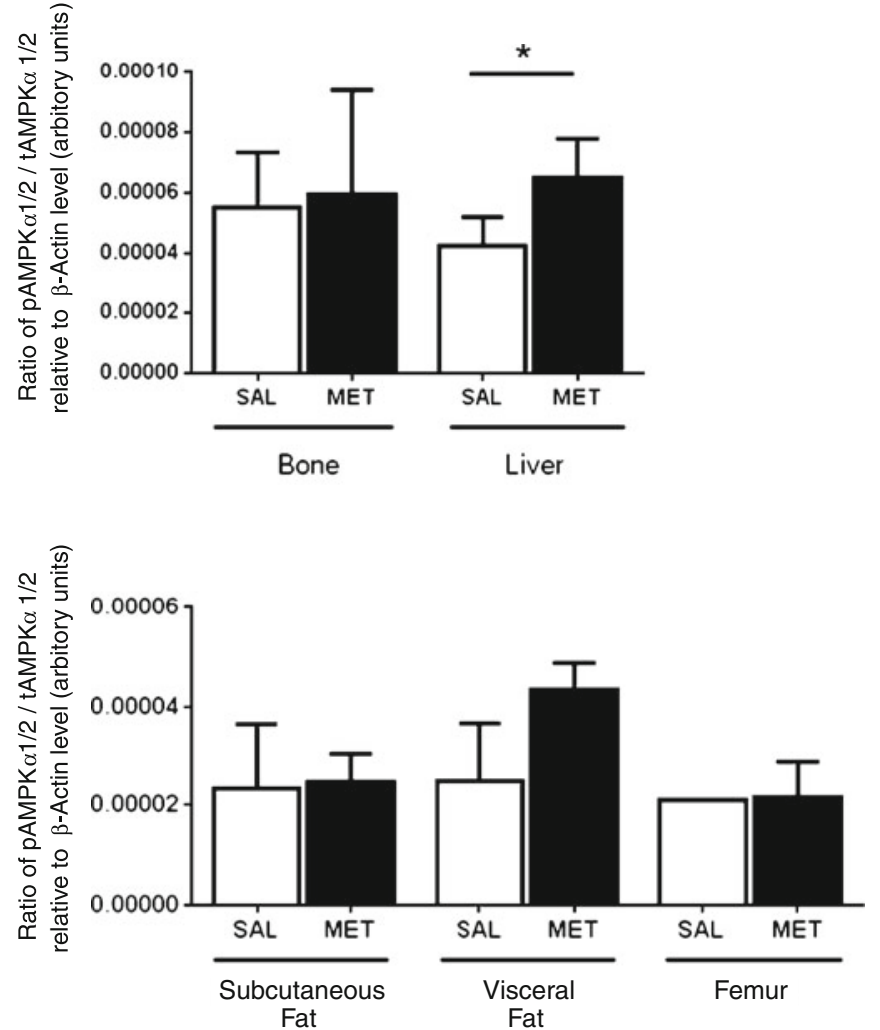

mice treated with metformin $(100 \mathrm{mg} / \mathrm{kg})$ for 1 month. Representative immunoblots are shown, repeated with similar results twice. $\mathbf{b}$, ii Blots were quantified using image $J$ and the pAMPK to tAMPK ratio relative to $\beta$-actin was determined for each experiment. Bars represent mean \pm $\mathrm{SD}, n=4$ biological samples. c Results show expression of Osterix and runt related transcription factor 2 (Runx 2 ) normalised to $18 \mathrm{~s}$ rRNA in femora of saline and metformin groups after 1 month of treatment. Two separate RNA extractions were performed for each treatment group, each time RNA being pooled from three femora

WT and OVX mice, respectively. The absence of association between metformin treatment and AMPK activation in bone in vivo may suggest that metformin's effect on bone could be more relevant in the context of diabetes and primarily indirect by reducing the inflammatory state, the accumulation of advanced glycation end-products (AGEs) and the formation of reactive oxygen species (ROS).

We show for the first time that metformin, at the dose given, has no effect on fracture healing in a model of mid-diaphyseal transverse osteotomy in rats. We evaluated the effect of metformin 4 weeks after fracture to examine the endochondral ossification process, and our data show no effect of metformin on callus size or on the speed of the healing process. Diabetes 
mellitus has been associated with impaired fracture healing, mainly due to suppressed osteoblastogenesis caused by low expression of genes that control osteoblast differentiation [48-53]. Both intramembranous and endochondral ossification are impaired and diabetic bone shows delayed bone regeneration [53]. The effects of anti-diabetic drugs on fracture healing have not been extensively studied. Molinuevo et al. [9] have found that metformin treatment stimulates bone lesion regeneration in a defect model in parietal bone in control and diabetic rats. Similarly, Sedlinsky et al. [14] have shown, in a similar minimal lesion defect in rats, that metformin treatment increases the reossification of this small lesion while rosiglitazone impaired it. Interestingly, metformin increased TRAP activity in these parietal bone lesions, a marker of osteoclast activity. Our data suggest that metformin does not affect the endochondral ossification process, but we cannot exclude that metformin could have an effect on the remodelling of the fracture callus and its mechanical strength.

Our study has several limitations, including the use of a single dose of metformin and the fact that we did not investigate the impact of T2DM on the skeletal effect of metformin. Nevertheless, it strongly indicates that metformin does not promote bone formation or fracture repair in non-diabetic rodent models, in contrast to the increased osteogenesis shown in several in vitro and in vivo studies. This suggests that, similarly to what was shown for TZDs, the skeletal effects of metformin are not always observed and could vary depending on factors such as the strain/sub-strain of rodents, gender, age, dose and duration of treatment as well as the hormonal and the inflammatory states.

Acknowledgements This work has been supported by the Wellcome Trust grant (Grant Reference 086630) and a joint exchange grant between the Royal Society and CNRS (Centre national de la recherche scientifique) in France, as well as by the Society for Endocrinology.

\section{Conflicts of Interest None.}

Open Access This article is distributed under the terms of the Creative Commons Attribution Noncommercial License which permits any noncommercial use, distribution, and reproduction in any medium, provided the original author(s) and the source are credited.

\section{References}

1. Cheng AY, Fantus IG (2005) Oral antihyperglycemic therapy for type 2 diabetes mellitus. CMAJ 172:213-226

2. Grey A, Bolland M, Gamble G, Wattie D, Horne A, Davidson J, Reid IR (2007) The peroxisome proliferator-activated receptorgamma agonist rosiglitazone decreases bone formation and bone mineral density in healthy postmenopausal women: a randomized, controlled trial. J Clin Endocrinol Metab 92:1305-1310

3. Lecka-Czernik B (2010) Bone loss in diabetes: use of antidiabetic thiazolidinediones and secondary osteoporosis. Curr Osteoporos Rep 8:178-184
4. Cortizo AM, Sedlinsky C, McCarthy AD, Blanco A, Schurman L (2006) Osteogenic actions of the anti-diabetic drug metformin on osteoblasts in culture. Eur J Pharmacol 536:38-46

5. Kanazawa I, Yamaguchi T, Yano S, Yamauchi M, Sugimoto $T$ (2008) Metformin enhances the differentiation and mineralization of osteoblastic MC3T3-E1 cells via AMP kinase activation as well as eNOS and BMP-2 expression. Biochem Biophys Res Commun 375:414-419

6. Jang WG, Kim EJ, Bae IH, Lee KN, Kim YD, Kim DK, Kim SH, Lee CH, Franceschi RT, Choi HS, Koh JT (2011) Metformin induces osteoblast differentiation via orphan nuclear receptor SHP-mediated transactivation of Runx2. Bone 48:885-893

7. Shah M, Kola B, Bataveljic A, Arnett TR, Viollet B, Saxon L, Korbonits M, Chenu C (2010) AMP-activated protein kinase (AMPK) activation regulates in vitro bone formation and bone mass. Bone 47:309-319

8. Zhen D, Chen Y, Tang X (2010) Metformin reverses the deleterious effects of high glucose on osteoblast function. J Diabetes Complications 24:334-344

9. Molinuevo MS, Schurman L, McCarthy AD, Cortizo AM, Tolosa MJ, Gangoiti MV, Arnol V, Sedlinsky C (2010) Effect of metformin on bone marrow progenitor cell differentiation: in vivo and in vitro studies. J Bone Miner Res 25:211-221

10. Wu W, Ye Z, Zhou Y, Tan WS (2011) AICAR, a small chemical molecule, primes osteogenic differentiation of adult mesenchymal stem cells. Int J Artif Organs 34:1128-1136

11. Kasai T, Bandow K, Suzuki H, Chiba N, Kakimoto K, Ohnishi T, Kawamoto S, Nagaoka E, Matsuguchi T (2009) Osteoblast differentiation is functionally associated with decreased AMP kinase activity. J Cell Physiol 221:740-749

12. Gao Y, Li Y, Xue J, Jia Y, Hu J (2010) Effect of the anti-diabetic drug metformin on bone mass in ovariectomized rats. Eur $\mathrm{J}$ Pharmacol 635:231-236

13. Mai QG, Zhang ZM, Xu S, Lu M, Zhou RP, Zhao L, Jia CH, Wen ZH, Jin DD, Bai XC (2011) Metformin stimulates osteoprotegerin and reduces RANKL expression in osteoblasts and ovariectomized rats. J Cell Biochem 112:2902-2909

14. Sedlinsky C, Molinuevo MS, Cortizo AM, Tolosa MJ, Felice JI, Sbaraglini ML, Schurman L, McCarthy AD (2011) Metformin prevents anti-osteogenic in vivo and ex vivo effects of rosiglitazone in rats. Eur J Pharmacol 668:477-485

15. Wang C, Li H, Chen SG, He JW, Sheng CJ, Cheng XY, Qu S, Wang KS, Lu ML, Yu YC (2012) The skeletal effects of thiazolidinedione and metformin on insulin-resistant mice. J Bone Miner Metab 30:630-637

16. Vestergaard P, Rejnmark L, Mosekilde L (2005) Relative fracture risk in patients with diabetes mellitus, and the impact of insulin and oral antidiabetic medication on relative fracture risk. Diabetologia 48:1292-1299

17. Home PD, Pocock SJ, Beck-Nielsen H, Curtis PS, Gomis R, Hanefeld M, Jones NP, Komajda M, McMurray JJ (2009) Rosiglitazone evaluated for cardiovascular outcomes in oral agent combination therapy for type 2 diabetes (RECORD): a multicentre, randomised, open-label trial. Lancet 373:2125-2135

18. Kahn SE, Zinman B, Lachin JM, Haffner SM, Herman WH, Holman RR, Kravitz BG, Yu D, Heise MA, Aftring RP, Viberti G (2008) Rosiglitazone-associated fractures in type 2 diabetes: an analysis from A Diabetes Outcome Progression Trial (ADOPT). Diabetes Care 31:845-851

19. Mancini T, Mazziotti G, Doga M, Carpinteri R, Simetovic N, Vescovi PP, Giustina A (2009) Vertebral fractures in males with type 2 diabetes treated with rosiglitazone. Bone 45:784-788

20. Tzoulaki I, Molokhia M, Curcin V, Little MP, Millett CJ, Ng A, Hughes RI, Khunti K, Wilkins MR, Majeed A, Elliott P (2009) Risk of cardiovascular disease and all cause mortality among patients with type 2 diabetes prescribed oral antidiabetes drugs: 
retrospective cohort study using UK general practice research database. BMJ 339:b4731

21. Zinmam B, Haffner SM, Herman WH, Holman RR, Lachin JM, Kravitz BG, Paul G, Jones NP, Aftring RP, Viberti G, Kahn SE, and the A Diabetes Outcome Progression Trial Study Group (2010) Effect of rosiglitazone, metformin and glyburide on bone biomarkers in patients with type 2 diabetes. J Clin Endocrinol Metab 95:134-142

22. Burt-Pichat B, Lafage-Proust MH, Duboeuf F, Laroche N, Itzstein C, Vico L, Delmas PD, Chenu C (2005) Dramatic decrease of innervation density in bone after ovariectomy. Endocrinology 146:503-510

23. Jeyabalan J, Shah M, Viollet B, Roux JP, Chavassieux P, Korbonits M, Chenu C (2012) Mice lacking AMP-activated protein kinase (AMPK)-alpha 1 catalytic subunit have increased bone remodeling and modified skeletal responses to hormonal challenges induced by ovariectomy and intermittent PTH treatment. J Endocrinol 214:349-358

24. Harrison LJ, Cunningham JL, Stromberg L, Goodship AE (2003) Controlled induction of a pseudarthrosis: a study using a rodent model. J Orthop Trauma 17:11-21

25. Amanat N, McDonald M, Godfrey C, Bilston L, Little D (2007) Optimal timing of a single dose of zoledronic acid to increase strength in rat fracture repair. J Bone Miner Res 22:867-876

26. Chappard D, Palle S, Alexandre C, Vico L, Riffat G (1987) Bone embedding in pure methyl methacrylate at low temperature preserves enzyme activities. Acta Histochem 81:183-190

27. Chavassieux PM, Arlot ME, Reda C, Wei L, Yates AJ, Meunier PJ (1997) Histomorphometric assessment of the long-term effects of alendronate on bone quality and remodeling in patients with osteoporosis. J Clin Invest 100:1475-1480

28. Parfitt AM, Drezner MK, Glorieux FH, Kanis JA, Malluche H, Meunier PJ, Ott SM, Recker RR (1987) Bone histomorphometry: standardization of nomenclature, symbols, and units. Report of the ASBMR Histomorphometry Nomenclature Committee. J Bone Miner Res 2:595-610

29. Zaman G, Sunters A, Galea GL, Javaheri B, Saxon LK, Moustafa A, Armstrong VJ, Price JS, Lanyon LE (2012) Loading-related regulation of transcription factor EGR2/Krox-20 in bone cells is ERK1/2 proteinmediated and prostaglandin, Wnt signaling pathway-, and insulin-like growth factor-1 axis-dependent. J Biol Chem 287:3946-3962

30. Amini H, Ahmadiani A, Gazerani P (2005) Determination of metformin in human plasma by high-performance liquid chromatography. J Chromatogr B Analyt Technol Biomed Life Sci 824:319-322

31. Kaneb HM, Sharp PS, Rahmani-Kondori N, Wells DJ (2011) Metformin treatment has no beneficial effect in a dose-response survival study in the SOD1(G93A) mouse model of ALS and is harmful in female mice. PLoS One 6:e24189

32. Fryer LG, Parbu-Patel A, Carling D (2002) The anti-diabetic drugs rosiglitazone and metformin stimulate AMP-activated protein kinase through distinct signaling pathways. J Biol Chem 277:25226-25232

33. Janghorbani M, Van Dam RM, Willett WC, Hu FB (2007) Systematic review of type 1 and type 2 diabetes mellitus and risk of fracture. Am J Epidemiol 166:495-505

34. Yamamoto M, Yamaguchi T, Yamauchi M, Kaji H, Sugimoto T (2009) Diabetic patients have an increased risk of vertebral fractures independent of BMD or diabetic complications. J Bone Miner Res 24:702-709

35. Oner G, Ozcelik B, Ozgun MT, Ozturk F (2011) The effects of metformin and letrozole on endometrium and ovary in a rat model. Gynecol Endocrinol 27:1084-1086

36. Wang XF, Zhang JY, Li L, Zhao XY, Tao HL, Zhang L (2011) Metformin improves cardiac function in rats via activation of AMPactivated protein kinase. Clin Exp Pharmacol Physiol 38:94-101

37. Souza-Mello V, Gregorio BM, Cardoso-de-Lemos FS, de Carvalho L, Aguila MB, Mandarim-de-Lacerda CA (2010) Comparative effects of telmisartan, sitagliptin and metformin alone or in combination on obesity, insulin resistance, and liver and pancreas remodelling in C57BL/6 mice fed on a very high-fat diet. Clin Sci (Lond) 119:239-250

38. Ackert-Bicknell CL, Shockley KR, Horton LG, Lecka-Czernik B, Churchill GA, Rosen CJ (2009) Strain-specific effects of rosiglitazone on bone mass, body composition, and serum insulinlike growth factor-I. Endocrinology 150:1330-1340

39. Jeyabalan J, Shah M, Viollet B, Chenu C (2012) AMP-activated protein kinase pathway and bone metabolism. J Endocrinol 212:277-290

40. Bak EJ, Park HG, Kim M, Kim SW, Kim S, Choi SH, Cha JH, Yoo YJ (2010) The effect of metformin on alveolar bone in ligature-induced periodontitis in rats: a pilot study. J Periodontol 81:412-419

41. Liu L, Zhang C, Hu Y, Peng B (2012) Protective effect of metformin on periapical lesions in rats by decreasing the ratio of receptor activator of nuclear factor kappa B ligand/osteoprotegerin. J Endod 38:943-947

42. Berlie HD, Garwood CL (2010) Diabetes medications related to an increased risk of falls and fall-related morbidity in the elderly. Ann Pharmacother 44:712-717

43. Loke YK, Singh S, Furberg CD (2009) Long-term use of thiazolidinediones and fractures in type 2 diabetes: a metaanalysis. CMAJ 180:32-39

44. Monami M, Cresci B, Colombini A, Pala L, Balzi D, Gori F, Chiasserini V, Marchionni N, Rotella CM, Mannucci E (2008) Bone fractures and hypoglycemic treatment in type 2 diabetic patients: a case-control study. Diabetes Care 31:199-203

45. Borges JL, Bilezikian JP, Jones-Leone AR, Acusta AP, Ambery PD, Nino AJ, Grosse M, Fitzpatrick LA, Cobitz AR (2011) A randomized, parallel group, double-blind, multicentre study comparing the efficacy and safety of Avandamet (rosiglitazone/metformin) and metformin on long-term glycaemic control and bone mineral density after 80 weeks of treatment in drug-naive type 2 diabetes mellitus patients. Diabetes Obes Metab 13:1036-1046

46. van Lierop AH, Hamdy NA, van der Meer RW, Jonker JT, Lamb HJ, Rijzewijk LJ, Diamant M, Romijn JA, Smit JW, Papapoulos SE (2012) Distinct effects of pioglitazone and metformin on circulating sclerostin and biochemical markers of bone turnover in men with type 2 diabetes mellitus. Eur J Endocrinol 166:711-716

47. Zhou G, Myers R, Li Y, Chen Y, Shen X, Fenyk-Melody J, Wu M, Ventre J, Doebber T, Fujii N, Musi N, Hirshman MF, Goodyear LJ, Moller DE (2001) Role of AMP-activated protein kinase in mechanism of metformin action. J Clin Invest 108:1167-1174

48. Zhou Loder RT (1988) The influence of diabetes mellitus on the healing of closed fractures. Clin Orthop Relat Res 232:210-216

49. Chaudhary SB, Liporace FA, Gandhi A, Donley BG, Pinzur MS, Lin SS (2008) Complications of ankle fracture in patients with diabetes. J Am Acad Orthop Surg 16:159-170

50. Hamann C, Goettsch C, Mettelsiefen J, Henkenjohann V, Rauner M, Hempel U, Bernhardt R, Fratzl-Zelman N, Roschger P, Rammelt S, Gunther KP, Hofbauer LC (2011) Delayed bone regeneration and low bone mass in a rat model of insulin-resistant type 2 diabetes mellitus is due to impaired osteoblast function. Am J Physiol Endocrinol Metab 301:E1220-E1228

51. Ogasawara A, Nakajima A, Nakajima F, Goto K, Yamazaki M (2008) Molecular basis for affected cartilage formation and bone union in fracture healing of the streptozotocin-induced diabetic rat. Bone 43:832-839

52. Retzepi M, Donos N (2010) The effect of diabetes mellitus on osseous healing. Clin Oral Implants Res 21:673-681

53. Hamann C, Kirschner S, Gunther KP, Hofbauer LC (2012) Bone, sweet bone- osteoporotic fractures in diabetes mellitus. Nat Rev Endocrinol 8:297-305 